\title{
Hospitalizations associated with salmonellosis among seniors in Canada, 2000-2010
}

\author{
P. TURGEON ${ }^{1 *}$, R. MURRAY ${ }^{2}$ AND A. NESBITT ${ }^{2}$ \\ ${ }^{1}$ National Microbiology Laboratory at Saint-Hyacinthe, Public Health Agency of Canada, Saint-Hyacinthe, \\ Canada \\ ${ }^{2}$ Centre for Food-borne, Environmental and Zoonotic Infectious Diseases Public Health Agency of Canada, \\ Guelph, Canada
}

Received 3 March 2016; Final revision 20 December 2016; Accepted 26 January 2017; first published online 23 February 2017

\section{SUMMARY}

Salmonella is one of the most prevalent bacteria associated with enteric illness in Canada and seniors are considered a vulnerable population more likely to develop severe illness. In the coming decades, hospitalizations and deaths associated with Salmonella in seniors could represent a challenge due to an aging population in Canada. The numbers of non-typhoidal (NT) Salmonella-related hospitalizations from the Canadian Hospitalization Morbidity Database were analysed for a period of 10 years for seniors. Hospitalization rate calculations and descriptive analyses were performed on variables associated with the burden of hospitalization and compared with the adult age group. Estimates of hospitalizations and deaths associated with domestically acquired Salmonella (accounting for under-reporting) were also calculated. This study found that $50 \%$ of the NT Salmonella-related hospitalization and $82 \%$ of the deaths recorded in the Canadian adult population occurred in seniors. The length of hospitalization stay was also longer in seniors ( 7 days) than other adults (4 days). We estimated that each year, 535 hospitalizations and 27 deaths are related to domestically acquired Salmonella in seniors. Senior populations represent a substantial percentage of Salmonella-related hospitalizations and deaths in Canada and the burden associated with those hospitalizations is also greater. This should be considered when developing estimates of medical costs and implementing prevention activities.

Key words: Burden of illness, hospitalization, salmonella, seniors.

\section{INTRODUCTION}

Non-typhoidal (NT) Salmonella infections remain an important public health problem in Canada and worldwide [1]. In Canada, NT Salmonella is one of the leading bacterial causes of enteric illness with more than 100000 domestically acquired estimated

\footnotetext{
* Author for correspondence: P. Turgeon, National Microbiology Laboratory at Saint-Hyacinthe, Public Health Agency of Canada, C.P. 5000, Saint-Hyacinthe, Quebec, Canada J2S 7C6.

(Email: patricia.turgeon@canada.ca)
}

cases each year [2]. Of these, $80 \%$ are estimated to be of foodborne origin. NT Salmonella is also the leading pathogen for the estimated annual cost of illness and quality-adjusted life years (QALY) loss in the United States, with an estimated cost of $3 \cdot 3$ billion and 17000 QALYs [3]. Although most cases of NT Salmonella are self-limiting, some will lead to severe symptoms and sometimes to severe invasive infections, including bacteraemia and arthritis [4]. The economic burden of NT Salmonella is substantial and hospitalizations and deaths associated with severe infections account for much of this cost [3]. Some 
groups of individuals are considered more at risk of severe NT Salmonella infection, including young children, pregnant woman, immunocompromised individuals and seniors [5]. Among those groups, seniors are one sub-population that is expected to change significantly in the next decade. With increasing lifespan and a demographic shift towards a larger proportion of seniors, Statistics Canada expects that people over 60 , who represented $20 \%$ of the Canadian population in 2011, will increase to $30 \%$ by 2035 - an increase of $56 \%$ for this age group or more than 5 million people [6]. Moreover, the proportion of people over 80 will also increase over time, meaning that they will come to represent a larger percentage of the senior population. This is noteworthy since in Canada the health-adjusted life expectancy the average number of healthy years that a person lives - is $<72$ years [7], which means that even if people live longer, they are not necessarily living healthy.

In older people several factors can contribute to more severe consequences following infection by enteric pathogens, including NT Salmonella. Aging is associated with changes and deterioration of the immune system. In older adults, adaptive immune function decreases as functional alterations in macrophages and granulocytes occur [8]. Moreover, a natural shift to memory T-cells reduces the efficiency of the cell-mediated response when new exposures to pathogens occur $[8,9]$. Various other factors may also influence immunity in older people, including therapies associated with chronic diseases (e.g. rheumatoid arthritis, cancer) and malnutrition [1012]. Aging is also associated with a higher chronic disease incidence and it has been reported that hospitalized salmonellosis patients with chronic diseases may be at increased risk for adverse outcomes, including death and longer length of stay [13].

The growth of the senior population represents an important challenge for the health care system in terms of capacity and expenditures. The incidence of reported cases of NT Salmonella in Canada is approximately $18 / 100000$ and $20 \%$ comes from seniors, but higher hospitalization and death rates have been reported for this age group [14, 15]. Better understanding of the actual burden of NT Salmonella-related hospitalizations in seniors is essential to be able to anticipate future burden. Therefore, the objectives of this study were to describe NT Salmonella-related hospitalization in seniors from 2000 to 2010 in Canada and generate estimates of hospitalizations associated with domestically acquired foodborne NT Salmonella in that vulnerable population.

\section{METHODS}

The numbers of NT Salmonella-related hospitalized cases were extracted from the Hospital Morbidity Database (HMDB) of the Canadian Institute for Health Information (CIHI) from 1 April 2000 to 31 March 2010. This database contains national discharge statistics from all acute care facilities across Canada; however, it does not include discharge data from chronic care, rehabilitation, psychiatric facilities, and emergency department visits [16, 17]. The HMDB contains clinical and demographic information on inpatients. Key variables include patient sex, patient age, province of residency, date of admission, date of discharge, discharge disposition (includes death outcomes), and length of stay. It also contains diagnosis codes from the International Statistical Classification of Diseases and Related Health Problems (ICD-9$\mathrm{CM}$ and ICD-10) published by the WHO (World Health Organization). For each discharge, 16-25 diagnostic codes were captured depending on the year. To ensure consistency in this study, a case was defined as a patient listed in the HMDB for whom one of the diagnosis codes associated with NT Salmonella (Table 1) had been recorded anywhere in the first 16 diagnostic code categories. The database also contains the variable 'most responsible diagnosis' for hospitalization, which is defined by the diagnosis or condition that can be described as being most responsible for the patient's stay in hospital. To be consistent with another Canadian study on hospitalizations, repeat visits with a diagnostic of NT Salmonella within 30 days were excluded as they were considered to be associated with the same episode [18]. Only patients for whom a Canadian province or territory was recorded as a place of residency were kept, thus excluding non-Canadian travelers hospitalized in Canada during the study period.

Total and annual numbers of hospitalizations were compiled for the 10 years of the study. The mean and annual hospitalization rates per 100000 were calculated for three age groups of adults: 20-59 years old, 60-79 years old, and 80 years and over. Population estimates from Statistics Canada for 2000-2010 were used for rate calculations [19]. The estimated percentage of reported cases hospitalized was calculated by dividing the numbers of hospitalized cases by the number of reported cases of Salmonella obtained 
Table 1. ICD-10 and ICD-9-CM codes for salmonellosis and selected chronic diseases

\begin{tabular}{lll}
\hline \hline & \multicolumn{1}{l}{ Codes } & \\
\cline { 2 - 3 } Disease & ICD-9-CM & ICD-10 \\
\hline Salmonellosis & & \\
$\quad$ Enteritis & $003 \cdot 0$ & $\mathrm{~A} 02 \cdot 0$ \\
Septicemia & $003 \cdot 1$ & $\mathrm{~A} 02 \cdot 1$ \\
$\quad$ Local infections & $003 \cdot 2$ & $\mathrm{~A} 02 \cdot 2$ \\
$\quad$ Other specified & $003 \cdot 8$ & $\mathrm{~A} 02 \cdot 8$ \\
$\quad$ Unspecified & $003 \cdot 9$ & $\mathrm{~A} 02 \cdot 9$ \\
Arthritis and rheumatism & $710 \cdot 0-739 \cdot 9$ (excl. 711, 728, 730) & $\mathrm{M} 05 \cdot 0-\mathrm{M} 99 \cdot 9(\mathrm{excl} . \mathrm{M} 60 \cdot 0-\mathrm{M} 63 \cdot 8, \mathrm{M} 86 \cdot 0-\mathrm{M} 87 \cdot 9)$ \\
Cardiovascular diseases & $393 \cdot 0-447 \cdot 0$ (excl. 415, 420, 421, 422) & $\mathrm{I} 05 \cdot 0-\mathrm{I} 02 \cdot 5, \mathrm{I} 27 \cdot 0-\mathrm{I} 28 \cdot 9, \mathrm{I} 31, \mathrm{I} 34 \cdot 0-\mathrm{I} 38$, \\
& & $\mathrm{I} 42-\mathrm{I} 45, \mathrm{I} 49 \cdot 0-\mathrm{I} 51 \cdot 9, \mathrm{I} 70 \cdot 0-\mathrm{I} 78 \cdot 9$ \\
Cancer & $140 \cdot 0-239 \cdot 2$ & $\mathrm{C} 00 \cdot 0-\mathrm{D} 48 \cdot 9$ \\
Diabetes & $249 \cdot 0-250 \cdot 9$ & $\mathrm{E} 10 \cdot 0-\mathrm{E} 14 \cdot 9$ \\
\hline \hline
\end{tabular}

from the Canadian Notifiable Disease Surveillance System [14]. Descriptive analyses (percentages, means, and medians) were performed on variables associated with the burden of hospitalization: length of stay and death. The discharge disposition variable in the database was used to identify deaths, meaning that all patients hospitalized with a diagnosis of NT Salmonella and who died at the hospital were recorded, reflecting both underlying and contributing causes of death. Medians were used when variable distributions were skewed. Descriptive analyses were also performed on comorbidity recorded according to the four most prevalent chronic diseases affecting seniors in Canada: arthritis and rheumatism, cardiovascular diseases, cancer, and diabetes [20] (Table 1). Diagnostic codes associated with these chronic diseases are presented in Table 1. Death outcomes and chronic disease status for hospitalizations with salmonellosis as the most responsible diagnosis (or the main reason for hospital stay) were also described. Statistical tests were used to test significance - the $\chi^{2}$ test was used for comparing proportions among age groups and the Wilcoxon rank-sum and KruskalWallis tests were used to test differences between medians. Data analyses were performed by using SAS statistical software version 9.3 [21].

Estimates of hospitalizations and deaths associated with total and domestically acquired foodborne NT Salmonella were calculated from the modeling approach used for the estimation of foodborne illnessrelated hospitalizations and deaths in Canada described in Thomas et al. [18]. This analysis accounted for under-reporting and under-diagnosis (adjusting for a multiplier of 1.8). The proportion of salmonellosis cases caused by foodborne transmission
$(80 \%)$ was assumed to be the same as in Thomas et al., and an age-specific proportion of travel-related illnesses was applied using data from FoodNet Canada (2005-2013), Canada's integrated enteric disease surveillance system based on sentinel sites [22] (Table 4). Monte Carlo simulations were performed to account for uncertainty generating mean estimates and $90 \%$ probability intervals (PI).

\section{RESULTS}

\section{Number of hospitalizations and rates}

From April 2000 to March 2010, 6148 NT Salmonellarelated hospital admissions were recorded in adults aged 20 years and over. Although seniors $(60+)$ represented $23 \%$ of reported NT Salmonella cases in adults in Canada during this time period, almost 50\% (2995) of NT Salmonella-related hospitalizations occurred in this age group. In seniors, NT Salmonella-related hospital admissions represent an estimated 32\% (2995/ 9634) of nationally reported cases, compared with $10 \%$ (3153/32 173) in other adults. Annual numbers and hospitalization rates are presented in Table 2. For each age group, hospitalization rates in men and women were not significantly different from each other. The diagnostic code Salmonella enteritis was the most recorded for each age group, followed by Salmonella sepsis and localized Salmonella infections when type of infection was specified (Table 3). Unspecified infections represented about $7 \%$ of hospitalizations in each age group. Proportions of hospitalizations where NT Salmonella diagnostic codes were captured as the most responsible diagnosis for hospitalization were the same $(68 \%)$, regardless of age group. 
Table 2. Annual numbers and hospitalization rates for salmonellosis (2000-2010)

\begin{tabular}{lllll}
\hline \hline $\begin{array}{l}\text { Age } \\
\text { group }\end{array}$ & $\begin{array}{l}\text { Population } \\
(2006)\end{array}$ & $\begin{array}{l}\text { Population } \\
(2006-\% \text { adult) }\end{array}$ & $\begin{array}{l}\text { Hospitalizations } \\
(\text { mean by year) }(95 \% \mathrm{CI})\end{array}$ & $\begin{array}{l}\text { Hospitalization incidence } \\
(/ 100000)(95 \% \mathrm{CI})\end{array}$ \\
\hline $20-59$ & 18791155 & 76 & $296(261-331)$ & $1 \cdot 7(1 \cdot 5-1 \cdot 8)$ \\
$60-79$ & 4762501 & 19 & $207(179-235)$ & $4 \cdot 3(3 \cdot 7-4 \cdot 9)$ \\
$80+$ & 1150726 & $0 \cdot 05$ & $80(64-96)$ & $6 \cdot 9(5 \cdot 5-8 \cdot 4)$ \\
\hline \hline
\end{tabular}

* Percentage of the Canadian adult (20+) population.

Table 3. Percentages of hospitalizations, deaths and length of stay according to NT Salmonella as the most responsible diagnostic codes (2000-2010)

\begin{tabular}{llll}
\hline \hline & $\%$ & $\%$ & $\begin{array}{l}\text { Length of stay } \\
\text { Age groups }\end{array}$ \\
\hline Hospitalization* & $\begin{array}{l}\text { Died } \\
\text { (days-median) }\end{array}$ \\
All & & & \\
Enteritis & $74 \cdot 3 \dagger$ & $0 \cdot 5$ & 4 \\
Septicemia & $12 \cdot 8$ & $0 \cdot 3 *$ & 4 \\
Local infections & $1 \cdot 5$ & $2 \cdot 5$ & 5 \\
60-79 & & 0 & 5 \\
All & & & \\
Enteritis & $77 \cdot 4$ & $2 \cdot 4$ & 6 \\
Septicemia & $12 \cdot 4$ & $1 \cdot 5$ & 5 \\
Local infections & $1 \cdot 4$ & $9 \cdot 2$ & 9 \\
80+ & & 5 & 7 \\
All & & & \\
Enteritis & 77 & $5 \cdot 3$ & 9 \\
Septicemia & $13 \cdot 3$ & $3 \cdot 5$ & 7 \\
Local infections & $0 \cdot 7$ & $16 \cdot 2$ & 11 \\
All adults & & 0 & 7 \\
All & & & \\
Enteritis & $75 \cdot 7$ & $1 \cdot 77$ & 5 \\
Septicemia & $12 \cdot 8$ & 4 & 7 \\
Local infections & $1 \cdot 4$ & $0 \cdot 2$ & 6 \\
\hline
\end{tabular}

* Total not equal to 100 because unspecified and other infections not included.

$\dagger$ Enteritis represents $74 \cdot 3 \%$ of NT Salmonella-related hospitalization among the $20-59$ age group.

$\$$ The $0 \cdot 3 \%$ of patients with identified enteritis has the most responsible diagnostic in this age group who died at the hospital.

\section{Length of stay and outcome}

The median length of stay for NT Salmonella-related hospitalization in seniors was 7 days compared with 4 days for other adults. The length of stay also increased $(P<0.05)$ with age with a median length of stay of 6 days for people aged 60-79 years and 9 days for people aged 80 years and over. Moreover, median days in hospital varied depending on type of infections contracted, with longer stays associated with sepsis (Table 3).
When looking at hospitalizations when NT Salmonella was identified as one of the diagnostic codes, deaths within hospital increased with age $(P<$ 0.05 ) for seniors. Deaths occurred in $4.3 \%$ of patients aged $60-79$ years and in $7 \cdot 4 \%$ of patients aged 80 years and over, for a mean percentage of deaths of $5.2 \%$ in seniors, representing $82 \%$ of the deaths recorded in the adult population. In other adults, $1 \cdot 1 \%$ of patients with salmonellosis died at the hospital. Similarly, deaths at the hospital increased with age $(P<0.05)$ when NT Salmonella was recorded as the most responsible diagnosis; death occurred in $2 \cdot 4 \%$ of patients aged $60-79$ years and in $5.3 \%$ of patients aged 80 years and over. In comparison, $0.5 \%$ of other adults died at the hospital when NT Salmonella was identified as the most responsible diagnosis for hospitalization (Table 3).

\section{Chronic diseases}

Among seniors (60+) hospitalized with NT Salmonella as the most responsible diagnosis, $60 \%$ were also diagnosed with at least one of the four most prevalent chronic diseases in seniors in Canada, namely cardiovascular diseases, diabetes, arthritis and rheumatism, and cancer (Fig. 1). Among those with at least one chronic disease, $36 \%$ were affected by two or more. Table 1 shows ICD diagnostic codes associated with chronic diseases. Among patients with only one chronic disease recorded, $45 \%$ of them recorded cardiovascular diseases. No statistically significant differences among these proportions were observed when looking at the two age groups among seniors (60-79 and $80+)$. When two or more chronic diseases were recorded, the most frequent combination was cardiovascular disease and diabetes, with $19 \%$ of patients affected with this combination of diseases.

\section{Estimates of domestic foodborne hospitalizations and deaths}

After accounting for under-reporting and underdiagnosis, the annual estimate of total NT Salmonella- 
Table 4. Annual estimate of NT Salmonella-related hospitalizations and deaths (2000-2010)

\begin{tabular}{|c|c|c|c|c|c|}
\hline \multirow[b]{2}{*}{ Age group } & \multirow[b]{2}{*}{$\begin{array}{l}\% \text { Travel related } \\
(95 \% \mathrm{CI})^{*}\end{array}$} & \multicolumn{2}{|c|}{$\begin{array}{l}\text { Estimated annual hospitalizations mean } \\
(90 \% \text { probability interval) }\end{array}$} & \multicolumn{2}{|c|}{$\begin{array}{l}\text { Estimated annual deaths mean } \\
(90 \% \text { probability interval })\end{array}$} \\
\hline & & Total & $\begin{array}{l}\text { Domestically acquired, } \\
\text { foodborne }\end{array}$ & Total & $\begin{array}{l}\text { Domestically acquired, } \\
\text { foodborne }\end{array}$ \\
\hline $60+$ & $21 \cdot 1(15 \cdot 4-27 \cdot 7) \dagger$ & $534(375-733)$ & $336(251-433)$ & $27(18-42)$ & $17(10-26)$ \\
\hline $60-79$ & $26 \cdot 1(18 \cdot 9-34 \cdot 4)$ & $382(268-511)$ & $225(155-307)$ & $16(9-25)$ & $9(5-15)$ \\
\hline $80+$ & $7 \cdot 8(2 \cdot 2-18 \cdot 9)$ & 152 (99-228) & 111 (71-168) & $11(5-21)$ & $8(4-15)$ \\
\hline
\end{tabular}

* Unpublished data FoodNet 2005-2013.

$\dagger$ Not used in calculation of domestically acquired, foodborne result for the 60+ age group (results for 60-79 and 80+ age group models were added to account for distribution of hospitalizations between the two age groups).

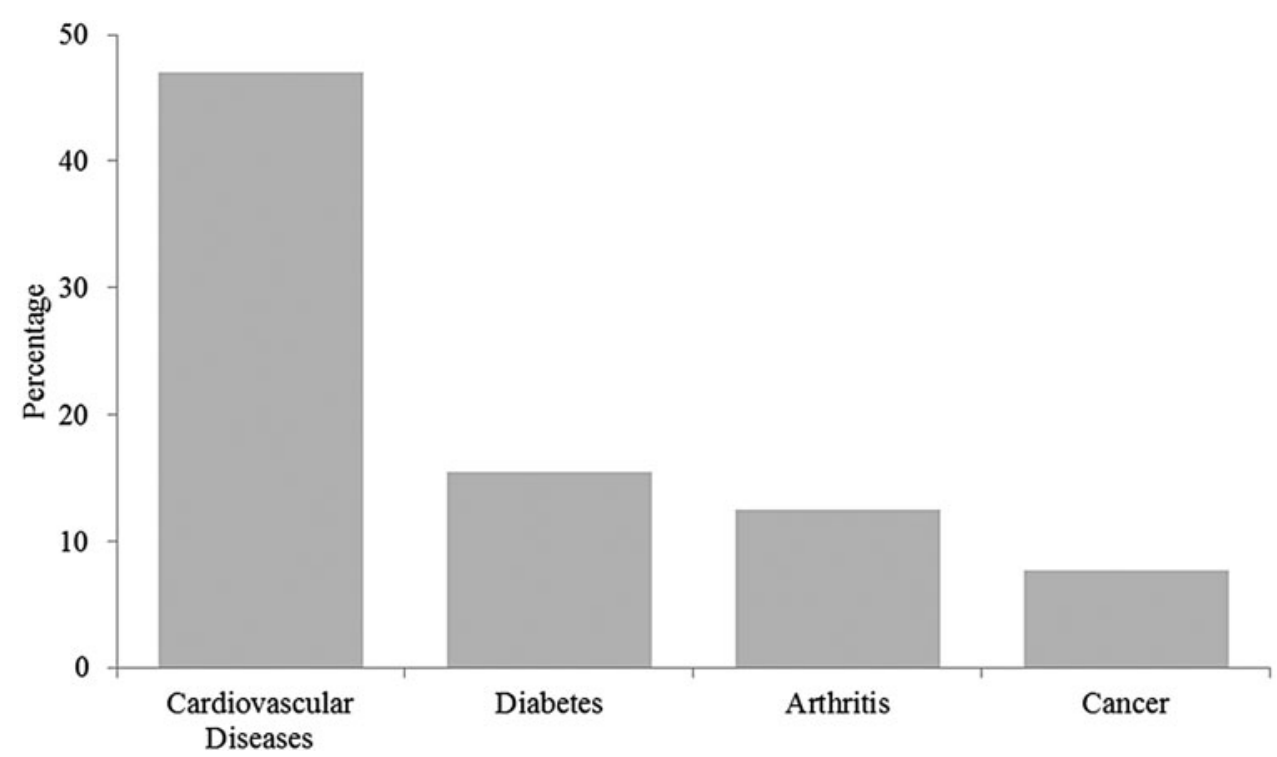

Fig. 1. Percentage of seniors diagnosed with chronic diseases and Salmonella as the most responsible diagnosis for hospitalization.

related hospitalizations in seniors in Canada is $534(90 \%$ PI 375-733) hospitalizations (Table 4), for an annual incidence of 11.2 hospitalizations per 100000 people, representing about $35 \%$ of Salmonella-related hospitalizations for all age groups [18]. A breakdown of the 6079 and 80 and over age groups can be found in Table 4 . When also accounting for the proportion of foodborne and travel-related infections (based on this age group), we estimated that each year, 336 (90\% PI 251-433) hospitalizations are related to domestically acquired foodborne NT Salmonella in seniors for an annual incidence of 5.7 hospitalizations per 100000 . The annual estimate of total NT Salmonella-related deaths in seniors is 27 (90\% PI 18-42) deaths; this includes both underlying and contributing causes of death, representing more than $90 \%$ of deaths estimated for all age groups. We also estimated that each year there are 17
(90\% PI 10-26) deaths related to domestically acquired foodborne NT Salmonella in seniors for an annual incidence of $0 \cdot 29$ deaths per 100000 .

\section{DISCUSSION}

Findings from this study showed that half of the NT Salmonella-related hospitalizations and most of the deaths recorded in the Canadian adult population occurred in seniors, even though they represent only a quarter of the adult population during the 10 years of the study. These proportions are of particular concern because they are expected to increase as the population aged 60 years and over is expected to increase by more than $56 \%$ by 2035 [6]. It is also important to mention that the hospitalization rate and the percentage of deaths within the hospital increased with 
age almost twofold among individuals aged 80 years and over compared with individuals aged between 60 and 79 years. This is noteworthy knowing that the proportion of people aged over 80 in the Canadian senior population is expected to increase over time. According to Statistics Canada, people aged over $80-20 \%$ of the Canadian senior population in 2011 - will increase to more than $25 \%$ of the seniors by 2035 , representing an increase of 1.8 million people for this age group. A similar finding was also identified in the USA, with higher proportions of the $80+$ age group hospitalized with NT Salmonella than the 65-79 age group [23].

This study also revealed that the burden associated with NT Salmonella-related hospitalization is greater as people get older. Seniors tend to stay longer in the hospital with a median length of stay that is $2-3$ days longer than other adults. This prolonged stay, especially of older people who tend to be affected by more than one medical condition, may contribute to an increased cost to the health care system. Results also showed that hospitalized seniors with NT Salmonella infection are more at risk of death than other adults hospitalized for the same condition. This was a similar finding identified in the USA for NT Salmonella, with a $2 \cdot 4 \%$ case fatality rate for the $65+$ age group compared with $0 \cdot 3 \%$ for 5-64 years [23]. Of the estimated annual costs attributed to Salmonella infections, fatal cases have been reported to have the highest cost per case in terms of medical care and loss of productivity [24].

Chronic dehydration, impaired immune function, existing chronic diseases and medication may make older people more vulnerable to infection and to more severe disease and health outcomes [25, 26].

A non-negligible proportion of seniors hospitalized with a NT Salmonella diagnosis as the most responsible diagnosis also had one or more underlying chronic diseases. Therefore, people affected by chronic disease may be more likely to seek medical care and be hospitalized when they have enteric symptoms. An American study also found a positive association between a higher number of chronic conditions in hospitalized salmonellosis patients and both disease severity and length of stay at the hospital [13]. Other studies have shown that chronic diseases influence the immune system in various ways that may lead to more severe illness for people suffering from bacterial infections, including salmonellosis [27, 28]. These results on chronic diseases in patients hospitalized with NT Salmonella remind us that elderly people afflicted with chronic diseases are functionally impaired and rely regularly on medication, which can make them even more susceptible to developing severe enteric diseases such as salmonellosis. This should be considered when prevention programs are developed and targeted to this sub-population.

Previous analyses of hospitalization data in Canada for all age groups revealed that Salmonella infections accounted for more hospitalizations than infections with any other bacterial foodborne pathogens during 2000-2010 [18]. Moreover, NT Salmonella was the second bacteria of importance when looking at the numbers of deaths. Of the total annual estimate of total domestically acquired Salmonella-related hospitalizations and deaths for all age groups in Canada, seniors represent about $35 \%$ of hospitalizations and more than $90 \%$ of deaths, highlighting the significant impact of the senior population on the burden of salmonellosis in Canada. These proportions are of particular concern as they are likely to be even greater in the future with the expected demographical shift towards a larger proportion of seniors and older seniors in the Canadian population in the coming decades [19]. Using a similar model, the USA estimated 5100 hospitalizations and 220 deaths each year related to NT Salmonella among adults aged 65+ [23]. This reflects a higher rate (13.7) per 100000 people than in Canada, which may reflect illness rates as well as their use of surveillance program data compared with the use of hospital administrative data to inform estimates. The use of linked surveillance data may capture more data on hospitalization outcomes for all laboratory-confirmed Salmonella cases, while administrative data may capture hospitalizations related to Salmonella that are first diagnosed at the hospital only.

Some limitations should be taken into account when looking at the results of this study. Hospitalization data are limited or not available through national surveillance programs. National level data for Canada is available through the databases of CIHI, however, NT Salmonella hospitalizations could have been under- or over-identified in those databases. According to a data quality analysis conducted by CIHI in 2009-2010 comparing the database and patient charts, $70 \%$ of admissions associated with intestinal infectious and parasitic diseases that were recorded in patient charts were identified in the database and 9\% of hospitalizations associated with those diseases identified in the database were not identified in the medical charts of the patients $[18,29]$. Because of under-reporting and under- 
diagnosis, numbers of hospitalizations are certainly underestimated. This is why calculated estimates presented in this study are important to integrate to better understand the true burden of hospitalization associated with salmonellosis in seniors. Limitations of the model to estimate domestically acquired hospitalizations and deaths are described in detail elsewhere [18], however, one additional limitation to note is the use of FoodNet Canada data for the age-specific proportion of travelrelated illness that may not be fully representative of the Canadian population [22]. Understanding cause of death is complex, especially when taking into account comorbidities; these results describe deaths of people who died after being infected with Salmonella and the resulting illness may be either the cause or the contributing cause of death.

This study describes specific characteristics of NT Salmonella-related hospitalization in seniors and therefore increases our understanding of the severity and health care use by age group. For the first time, age group-specific estimates of NT Salmonella-related hospitalizations and deaths in Canada were calculated, highlighting the importance of the senior population in the over-all burden estimates. This should be considered when developing direct and indirect medical costs to estimate total economic cost of the illness in Canada. NT Salmonella continues to be a common pathogen causing illness and older people are considered a vulnerable population to these infections and especially to severe outcomes. Because of their vulnerable status, identification of unique risk factors and targeted prevention with seniors should be pursued to reduce the number of salmonellosis cases and to reduce hospitalizations and deaths associated with this disease.

\section{ACKNOWLEDGEMENTS}

This study used data and information provided by the Canadian Institute for Health Information. However, the analyses, conclusions, opinions and statements expressed herein are those of the authors and not necessarily those of the Canadian Institute for Health Information. The authors thank the Data Coordination and Access Program of the Public Health Agency of Canada for their support with the database.

\section{DECLARATION OF INTEREST}

None.

\section{REFERENCES}

1. Majowicz SE, et al. The global burden of nontyphoidal Salmonella gastroenteritis. Clinical Infectious Diseases 2010; 50: 882-889.

2. Thomas MK, et al. Estimates of the burden of foodborne illness in Canada for 30 specified pathogens and unspecified agents, circa 2006. Foodborne Pathogens and Disease 2013; 10: 639-648.

3. Hoffmann S, Batz MB, Morris JGJ. Annual cost of illness and quality-adjusted life year losses in the United States due to 14 foodborne pathogens. Journal of Food Protection 2012; 75: 1292-1302.

4. Threlfall EJ, Wain J, Lane C. Salmonellosis. In: Palmer SR, et al., eds. Oxford Textbook of Zoonoses Biology, Clinical Practice, and Public Health Control, 2nd edn. New York, USA: Oxford University Press, 2011, pp. 252-271.

5. Gerba CP, Rose JB, Haas CN. Sensitive populations: who is at the greatest risk? International Journal of Food Microbiology 1996; 30: 113-123.

6. Statistics Canada. Projected population, by projection scenario, age and sex, as of July 1, Canada, provinces and territories. (http://www5.statcan.gc.ca/cansim/a26? lang=eng\&retrLang=eng\&id $=0520005 \&$ tabMode $=$ dataTable \&srchLan $=-0520001 \&$ p0520001 $=-0520001 \&$ p0520002=0520009). Accessed 14 July 2015.

7. Public Health Agency of Canada Steering Committee on Health-Adjusted Life Expectancy. Health-adjusted life expectancy in Canada: 2012 report from the Public Health Agency of Canada. Ottawa; 2012.

8. Khanna KV, Markham RB. A perspective on cellular immunity in the elderly. Clinical Infectious Diseases 1999; 28: 710-713.

9. Castle SC. Clinical relevance of age-related immune dysfunction. Clinical Infectious Diseases 2000; 31: 578-585.

10. Smith JL. Foodborne illness in the elderly. Journal of Food Protection 1998; 61: 1229-1239.

11. Klein NC, Go CH-U, Cunha BA. Infections associated with steroid use. Infectious Disease Clinics 2001; 15: 423-432.

12. Donskey CJ. The role of the intestinal tract as a reservoir and source for transmission of nosocomial pathogens. Clinical Infectious Diseases 2004; 39: 219-226.

13. Cummings PL., et al. Salmonellosis hospitalizations in the United States: associated chronic conditions, costs, and hospital outcomes, 2011, trends 200-2011. Foodborne Pathogens and Disease 2015; 13: 40-48.

14. Public Health Agency of Canada. Notifiable disease on-line. (http://dsol-smed.phac-aspc.gc.ca/dsol-smed/ ndis/index-eng.php). Accessed January 2015.

15. Kennedy M, et al. Hospitalizations and deaths due to Salmonella infections, FoodNet, 1996-1999. Clinical Infectious Diseases 2004; 38: S142-S148.

16. Canadian Institute for Health Information. Acute care. (https://www.cihi.ca/en/types-of-care/hospital-care/ acute-care). Accessed May 2016.

17. Canadian Institute for Health Information. Continuing care. (https://www.cihi.ca/en/types-of-care/hospital-care/ continuing-care). Accessed May 2016. 
18. Thomas MK, et al. Estimates of foodborne illnessrelated hospitalizations and deaths in Canada for 30 specified pathogens and unspecified agents. Foodborne Pathogens and Disease 2015; 12: 820-834.

19. Statistics Canada. Estimates of population by age and sex for Canada, provinces and territories. (http:// www23.statcan.gc.ca/imdb/p22SV.pl?Function= getSurvey\&lang $=$ en $\& \mathrm{db}=\mathrm{imdb} \& \mathrm{adm}=28 \& \mathrm{dis}=22 \&$ SDDS=3604). Accessed 7 January 2015.

20. Public Health Agency of Canada. The Chief Public Health Officer's Report on the State of Public Health in Canada 2010: Growing Older - Adding Life to Years. Ottawa: Government of Canada, 2010.

21. SAS-Institute-Inc. SAS 9·3. In. Cary, USA, 2003.

22. Public Health Agency of Canada. FoodNet Canada. Unpublished raw data. In, 2015.

23. Scallan E, et al. Bacterial enteric infections among older adults in the United States: foodborne diseases active surveillance network, 1996-2012. Foodborne Pathogens and Disease 2015; 12: 492-499.

24. Frenzen PD, et al. Salmonella cost estimate updated with FoodNet data. Food Review 1999; 22: 10-15.

25. Gavazzi G, Kraus K-H. Ageing and infection. Lancet Infectious Diseases 2002; 2: 659-666.

26. Warren JL, et al. The burden and outcomes associated with dehydration among US elderly, 1991. American Journal of Public Health 1994; 84: 1265-1269.

27. Lund BM, O'Brien SJ. The occurrence and prevention of foodborne disease in vulnerable people. Foodborne Pathogens and Disease 2011; 8: 961-973.

28. Kirkwood T. Mechanisms of ageing. In: Ebrahim S, Kalache A, eds. Epidemiology in Old Age. London, UK: BMJ Publishing Group, 1996, pp. 3-11.

29. Canadian Institute for Health Information. DAD 2009 2010 Re-abstraction Study. Ottawa, Canada: Canadian Institute for Health Information, 2012. 